\title{
Recurrent meningitis secondary to medically induced CSF leakage in the setting of a pituitary macroprolactinoma
}

Seifeldin Yahia, Simon Page, Nottingham University Hospital, Queen's Medical Centre, QMC,UK

\section{Contents}

A 49 year old gentleman was diagnosed with a pituitary macroprolactinoma at Leicester Royal Infirmary (LRI) (Baseline prolactin $8000 \mathrm{mU} / \mathrm{l}$ ). He was treated with Cabergoline $0.5 \mathrm{mg}$ twice/week. He developed cerebrospinal fluid (CSF) rhinorrhoea shortly after treatment was commenced and was listed for repair under the neurosurgical team at Queen's Medical Centre (QMC).

Six weeks later, while visiting Wales, he was admitted to Bangor hospital with headaches and vomiting. CSF cultures were positive for pneumococcus so he was treated with a 10 day course of Ceftriaxone. He continued on Cabergoline and this was associated with persistence of his CSF leak. He was discharged without prophylactic antibiotics with a plan for his CSF repair to be done 6/52 later at QMC in Nottingham. He was re-admitted to the LRI 3 weeks later with similar headaches and vomiting and was treated for recurrent meningitis with Meropenem despite negative cultures on CSF. He improved clinically, but he continued to experience a CSF leak. Two weeks later, he had a trans-sphenoidal repair of the sella using abdominal fat and fascia. This procedure was unsuccessful and 4 weeks later, he underwent a second endoscopic repair with a vascularized nasoseptal flap and was discharged with no further problems.

\begin{tabular}{|c|c|c|}
\hline Hospital & $\begin{array}{l}\text { Prolactin level (reference } \\
\text { range } 45 \text { - } 375 \mathrm{mU} / \mathrm{l})\end{array}$ & Time \\
\hline LRI & $8,000 \mathrm{mU} / \mathrm{I}$ & Baseline \\
\hline Bangor Hospital & $3,810 \mathrm{mU} / \mathrm{I}$ & One month on Cabergoline \\
\hline LRI & $1,500 \mathrm{mU} / \mathrm{l}$ & Three months on Cabergoline \\
\hline LRI & $882 \mathrm{mU} / \mathrm{l}$ & One Year on Cabergoline \\
\hline
\end{tabular}
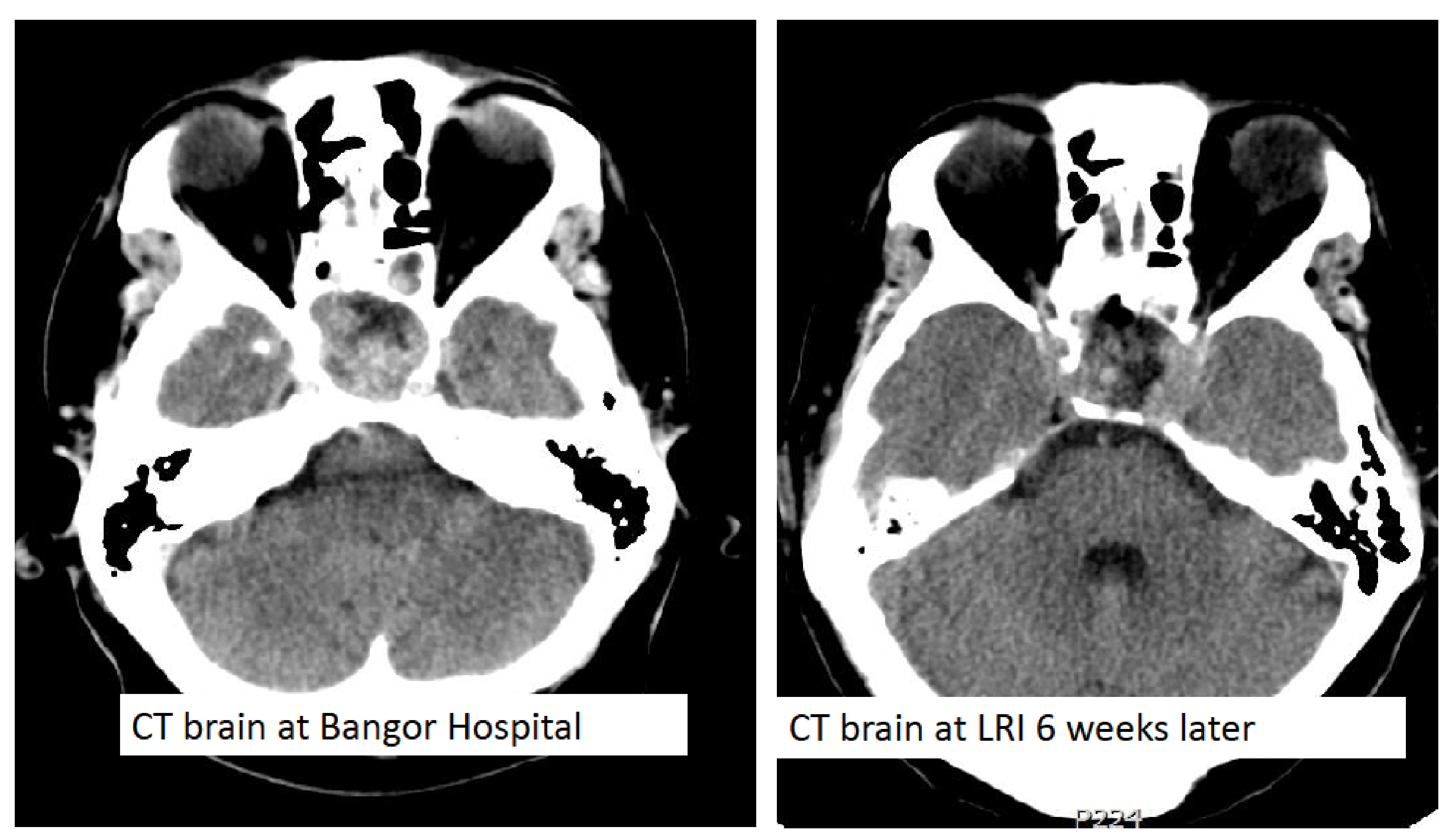

\section{Discussion:}

CSF rhinorrhoea may occur in the setting of pituitary adenomas, following a favourable response of invasive prolactinomas to initiation of dopamine agonist therapy. This risk might be even greater with cabergoline than with other dopamine agonists, due to the high sensitivity of prolactinomas to cabergoline. CSF leakage can occur within days of treatment and even minimal tumour shrinkage may lead to the development of a CSF fistula. Thus, we advise monitoring these patients closely and to actively forewarn them of this complication and the risk of life threatening meningitis. In this situation, prophylactic antibiotics to prevent meningitis remain controversial.

Surgical intervention via a trans-sphenoidal approach is the recommended initial treatment for definitive repair of the CSF leak. 